hep-th/0407150

LPTHE-04-17

\title{
Melvin Models and Diophantine Approximation
}

\author{
David Kutasov \\ EFI and Department of Physics \\ University of Chicago, Chicago, IL 6063\%, USA \\ Jens Marklof \\ School of Mathematics \\ University of Bristol, Bristol BS8 1TW, U.K. \\ Gregory W. Moore \\ Department of Physics, Rutgers University \\ Piscataway, NJ 08854-8019, USA
}

\begin{abstract}
Melvin models with irrational twist parameter provide an interesting example of conformal field theories with non-compact target space, and localized states which are arbitrarily close to being delocalized. We study the torus partition sum of these models, focusing on the properties of the regularized dimension of the space of localized states. We show that its behavior is related to interesting arithmetic properties of the twist parameter $\gamma$, such as the Lyapunov exponent. Moreover, for $\gamma$ in a set of measure one the regularized dimension is in fact not a well-defined number but must be considered as a random variable in a probability distribution.
\end{abstract}

July 16, 2004 


\section{Introduction}

Two dimensional conformal field theories (CFT's) corresponding to defects embedded in non-compact target spaces have many applications in string theory and are interesting in their own right [1],2].

As in scattering problems in quantum mechanics, the eigenstates of the Hamiltonian in such theories split into two classes. One consists of delta-function normalizable scattering states, which can propagate in the whole non-compact space. The other corresponds to normalizable states localized near the defect. In order to study the defect, one is particularly interested in the localized states and their interactions with the scattering states.

An example that has received some attention in recent years is orbifolds of flat noncompact space. In this case, the delocalized (scattering) states belong to the untwisted sector of the orbifold, while the localized ones are twisted sector states. For orbifolds by a finite group, the spectrum of localized states is discrete, with finite gaps between states. By contrast, orbifolds by infinite groups can have discrete but dense spectra of states. The latter case is particularly interesting since there is then no sharp distinction between localized and delocalized states.

More generally, while orbifolds by finite groups are well-studied, orbifolds by infinite groups introduce many new features, and have not been well-studied (some work has been done on time-dependent orbifolds; see [3] for a review). A better understanding of general CFT orbifolds by infinite groups might provide insights into string cosmology, the AdS/CFT correspondence, and noncommutative geometry [ [⿴囗十 .

In this note we will study an orbifold of $\mathbf{R} \times \mathbf{C}$ by the group $\mathbf{Z}$, known as the Melvin model, or the twisted circle. These models were introduced and studied in [5-10]. For further background on the Melvin model see [11,2] and references therein. We will see that as we vary the orbifold twist parameter, the model exhibits some unusual behavior, including divergences associated with a sum over almost delocalized twisted sector states. These divergences can be quantified using some results from the theory of Diophantine approximation. For background on Diophantine approximation see, e.g., [12-16].

The Melvin CFT is the orbifold

$$
(\mathbf{R} \times \mathbf{C}) / \mathbf{Z}
$$


where the generator $g$, of the group $\mathbf{Z}$ acts as

$$
\begin{aligned}
& y \rightarrow y+2 \pi R \\
& z \rightarrow e^{2 \pi i \gamma} z
\end{aligned}
$$

for $(y, z) \in \mathbf{R} \times \mathbf{C}$. When $\gamma$ is rational, e.g. $\gamma=1 / n$, one can think of the orbifold (1.2) as a $\mathbf{Z}_{n}$ orbifold of $S^{1} \times \mathbf{C}$. For irrational $\gamma$, it is not clear apriori whether (1.1) makes sense as a CFT (and string theory) background. One of our motivations below will be to explore this issue, by studying the torus partition sum of the theory. We will see that for irrational $\gamma$ the partition sum is very sensitive to the number theoretic properties of $\gamma$ (physical effects related to the arithmetic of irrational angles have appeared in some other recent investigations in string theory; see e.g. [17, 18, 19]).

We will mostly focus on the CFT $(\mathbf{R} \times \mathbf{C}) / \mathbf{Z}$. In string theory on $\mathbf{R}^{1,6} \times(\mathbf{R} \times \mathbf{C}) / \mathbf{Z}$ the consistency requirements for the existence of the theory are more stringent, and it is possible that the theory does not exist for irrational $\gamma$.

Although the orbifold (1.2) does not have fixed points, one can think of the origin of the $z$-plane as the location of a defect, near which the twisted states of the orbifold are localized. Indeed, consider a low lying state in the $w$-twisted sector. It winds $w$ times around the circle $\mathbf{R} / 2 \pi R \mathbf{Z}$ labelled by $y$. Its endpoints in the $z$-plane are separated by angle $2 \pi\|w \gamma\|$, where, for a real number $x,\|x\|$ denotes the distance to the nearest integer. 目

A classical string placed a distance $r$ from the origin has energy

$$
\alpha^{\prime 2} M^{2}(r)=(R w)^{2}+(r\|w \gamma\|)^{2}
$$

When $\|w \gamma\| \neq 0$, such winding strings are localized near the origin - their wavefunctions fall off exponentially as $r \rightarrow \infty$. The radial size of such $w$-twisted strings goes like $1 /\|w \gamma\|$. In fact, we see from (1.3) that strings stretched in the angular direction of the $z$-plane behave as if their effective tension is proportional to $\|w \gamma\|$; this will be important for our later discussion. After quantization, the reduced string tension is reflected in the presence of twisted oscillators for the worldsheet superfield $z$ with moding $\|w \gamma\|$.

For rational $\gamma$, the radial size $1 /\|w \gamma\|$ is bounded from above in the twisted sectors. Thus, there is a clear distinction between localized and delocalized sectors. When $\gamma$ is

1 Thus, defining the fractional part of $x,\{x\}=x-[x]$, one has $\|x\|=\min (\{x\}, 1-\{x\})$. 
irrational there are twisted sectors that are arbitrarily close to being delocalized, since $\|w \gamma\|$ is not bounded from below.

To study the theory for irrational $\gamma$, we would like to analyze the partition sum of the CFT (1.1) on a torus with modulus $q=e^{2 \pi i \tau}$; this corresponds to the trace of $q^{L_{0}-c / 24} \bar{q}^{\bar{L}_{0}-c / 24}$ over the eigenmodes of $\left(L_{0}, \bar{L}_{0}\right)$. For non-compact orbifolds, the trace over the untwisted sector is divergent - it is proportional to the volume of the target space. Sometimes, it is possible to regulate similar volume divergences by compactifying the space, but here this is not possible without breaking conformal invariance.

In [20] it was proposed, in a related context, to restrict the trace in the torus partition sum to the localized states, i.e. to the twisted sectors of the orbifold. This eliminates the usual volume divergence from the untwisted sector but, as we will see, leaves in some cases analogous divergences from "almost untwisted" sectors.

The partition sum of the localized states is given by

$$
Z_{\mathrm{loc}}(\tau ; \gamma):=\operatorname{Tr}_{\mathcal{H}_{\text {loc }}} q^{L_{0}-c / 24} \bar{q}^{\bar{L}_{0}-c / 24}
$$

where $\mathcal{H}_{\text {loc }}$ is a sum over twisted sectors

$$
\mathcal{H}_{\text {loc }}(\gamma):=\oplus_{\|w \gamma\| \neq 0} \mathcal{H}_{w}
$$

The partition sum $Z_{\text {loc }}(\tau ; \gamma)$ is not modular invariant. It transforms under $\tau \rightarrow-1 / \tau$ to the trace over the untwisted Hilbert space with a certain projection operator inserted. This is analogous to what happens for D-branes: the annulus amplitude, which can be thought of as a trace over open string states whose ends lie on the D-brane, is related by a modular transformation to a sum over closed strings that can be emitted by the D-brane.

By analogy to the D-brane case, it was proposed in 20] to study the regularized dimension of the space of localized states, which is given by $(\sqrt{1.4})$ in the limita $q \rightarrow 1$. For non-compact orbifolds by finite groups one finds in this limit

$$
Z_{\mathrm{loc}}(\tau \rightarrow 0 ; \gamma) \sim g_{c l}(\gamma) e^{\frac{\pi c}{6 \tau_{2}}}
$$

The leading exponential term in (1.6) is universal - it only depends on the central charge (or dimension of space). Thus, one can think of the quantity $g_{c l}$ as a measure of the density of localized states. Some properties of $g_{c l}$ for finite orbifold groups were described in [20].

${ }^{2}$ We set $\tau_{1}=0$, such that $q=e^{-2 \pi \tau_{2}}$, and take $\tau_{2} \rightarrow 0$. 
As we will see, in the irrational Melvin case the coefficient of the exponential in (1.6) behaves in an unusual way and does not have a good limit as $\tau_{2} \rightarrow 0$. First, it diverges like $\tau_{2}^{-b(\gamma)}$, with some constant $b(\gamma) \geq 1 / 2$. Moreover - and somewhat surprisingly - the coefficient of this divergence, while it is order 1 , does not have a well-defined limit as $\tau_{2} \rightarrow 0$ but varies as a random variable in a probability distribution. We explain this point, which is somewhat novel in conformal field theory, in sections 3.2, 3.3 below. A rigorous account is given in the appendix. One nice aspect of the discussion is that the behavior of the regularized dimension is related to the behavior of geodesics on a certain modular curve.

The regularized dimension of the space of localized states (1.6) is analogous to a similar regularized dimension which proved useful in RCFT [21]. The D-brane analog of $g_{c l}$ is the product of the tensions of the D-branes on which the open strings end (see, e.g., [22]). Note also that in models with spacetime fermions, the trace in (1.4) is usually taken to include a factor of $(-)^{F}$, such that spacetime fermions contribute with a minus sign, and there are usually large cancellations between bosons and fermions. For the purpose of estimating the high energy density of states, we should only sum over spacetime bosons (or over bosons plus fermions); see e.g. 23,24 for a discussion of the relevant issues.

In the remainder of this note we will study the behavior of the torus partition sum, and in particular of $g_{c l}$ (1.6), for irrational Melvin models. The main results are:

(1) When the twist $\gamma$ of the Melvin model is a Liouville number of a special kind, the one-loop partition function for bosons and fermions separately diverges for fixed $\tau$, although the string theory partition sum $Z_{B}-Z_{F}$ is finite. For such twists it is not clear that the Melvin conformal field theory makes sense. For $\gamma$ of Diophantine type this pathology is absent.

(2) The standard definition (1.6) of the regularized dimension determines not a number, but a random variable in a probability distribution. This is explained heuristically in sections 3.2 and 3.3. A rigorous discussion is given in the appendix.

(3) We can use the continued fraction approximations to $\gamma$ to define a modular invariant regulator in the case of irrational twists. We define a degree of delocalization and show that it is related to the Lyapunov exponent of $\gamma$. 


\section{Torus partition sum}

Using the definition of the Melvin CFT (1.1), (1.2), one can write the torus partition sum of the model. In the sector twisted by $g^{s}, s \in \mathbf{Z}(s \neq 0)$, one has: 3

$$
\begin{aligned}
& \operatorname{Tr}_{\mathcal{H}_{g} s} g^{t} q^{L_{0}-c / 24} \bar{q}^{\bar{L}_{0}-c / 24}= \\
& \operatorname{vol}(\mathbf{R})\left|\frac{\vartheta\left[\begin{array}{c}
\epsilon_{2} \\
\epsilon_{1}
\end{array}\right](0 \mid \tau)}{\eta^{3}}\right| \int_{-\infty}^{+\infty} \frac{d p}{2 \pi} q^{\frac{\alpha^{\prime}}{4}\left(p+s R / \alpha^{\prime}\right)^{2}} \bar{q}^{\frac{\alpha^{\prime}}{4}\left(p-s R / \alpha^{\prime}\right)^{2}} e^{2 \pi i(p R) t}\left|\frac{\vartheta\left[\begin{array}{c}
\epsilon_{2}+s \gamma \\
\epsilon_{1}+t \gamma
\end{array}\right](0 \mid \tau)}{\vartheta\left[\begin{array}{l}
\frac{1}{2}+s \gamma \\
\frac{1}{2}+t \gamma
\end{array}\right](0 \mid \tau)}\right|^{2}
\end{aligned}
$$

The $\left|\vartheta / \eta^{3}\right|$ prefactor is the contribution of the (bosonic and fermionic) oscillators on $\mathbf{R}$. $\epsilon_{1}, \epsilon_{2}=0, \frac{1}{2}$ label the spin structure of the fermions. The final ratio of theta functions is the partition function of the $N=2$ superfield twisted by $g^{s}$ and projected by $g^{t}$. Note that it only depends on the fractional parts $\{s \gamma\},\{t \gamma\}$.

In the orbifold theory we must sum over $g^{t}, t \in \mathbf{Z}$, to project onto invariant states, and divide by the order of the group $\mathbf{Z}$. We interpret

$$
\operatorname{vol}(\mathbf{R}) /|\mathbf{Z}|=2 \pi \mathrm{R}
$$

There is no factor of the volume of $\mathbf{C}$ because we are in a twisted sector. The net result is that the trace in the $g^{s}$ twisted sector in the orbifold theory is

$$
\begin{aligned}
& \operatorname{Tr}_{\mathcal{H}_{g} s} q^{L_{0}-c / 24} \bar{q}^{\bar{L}_{0}-c / 24}= \\
& 2 \pi R\left|\frac{\vartheta\left[\begin{array}{c}
\epsilon_{2} \\
\epsilon_{1}
\end{array}\right](0 \mid \tau)}{\eta^{3}}\right| \sum_{t \in \mathbf{Z}} \int_{-\infty}^{+\infty} \frac{d p}{2 \pi} q^{\frac{\alpha^{\prime}}{4}\left(p+s R / \alpha^{\prime}\right)^{2}} \bar{q}^{\frac{\alpha^{\prime}}{4}\left(p-s R / \alpha^{\prime}\right)^{2}} e^{2 \pi i(p R) t}\left|\frac{\vartheta\left[\begin{array}{c}
\epsilon_{2}+s \gamma \\
\epsilon_{1}+t \gamma
\end{array}\right](0 \mid \tau)}{\vartheta\left[\begin{array}{l}
\frac{1}{2}+s \gamma \\
\frac{1}{2}+t \gamma
\end{array}\right](0 \mid \tau)}\right|^{2} .
\end{aligned}
$$

In order to evaluate the $\tau \rightarrow 0$ asymptotics it is convenient to do the Gaussian integral over $p$ to get

$$
\sqrt{\frac{R^{2}}{\alpha^{\prime} \tau_{2}}}\left|\frac{\vartheta\left[\begin{array}{c}
\epsilon_{2} \\
\epsilon_{1}
\end{array}\right](0 \mid \tau)}{\eta^{3}}\right| \sum_{t \in \mathbf{Z}} e^{-\frac{\pi R^{2}}{\alpha^{\prime}} \frac{|t+s \tau|^{2}}{\tau_{2}}}\left|\frac{\vartheta\left[\begin{array}{c}
\epsilon_{2}+s \gamma \\
\epsilon_{1}+t \gamma
\end{array}\right](0 \mid \tau)}{\vartheta\left[\begin{array}{c}
\frac{1}{2}+s \gamma \\
\frac{1}{2}+t \gamma
\end{array}\right](0 \mid \tau)}\right|^{2}
$$

3 Our convention for theta functions is

$$
\frac{\vartheta\left[\begin{array}{l}
\theta \\
\phi
\end{array}\right](0 \mid \tau)}{\eta}=e^{2 \pi i \theta \phi} q^{\left(\frac{\theta^{2}}{2}-\frac{1}{24}\right)} \prod_{n=1}^{\infty}\left(1+e^{2 \pi i \phi} q^{n-\frac{1}{2}+\theta}\right)\left(1+e^{-2 \pi i \phi} q^{n-\frac{1}{2}-\theta}\right)
$$

where $\eta$ is the Dedekind eta function. 
Next we have to sum over the different twisted sectors and spin structures. The precise details of the sum depend on the particular theory - CFT on the orbifold, type 0 or type II string theory on $\mathbf{R}^{1,6}$ times the orbifold, etc; see e.g. [25] for a discussion. The different theories behave in a similar way as far as our analysis is concerned. To be concrete, consider type IIB string theory on $\mathbf{R}^{1,1} \times T^{5}$ times the orbifold. Here, $T^{5}$ is a five-torus of volume $V_{5}$; the compactification is convenient for studying the $\tau_{2} \rightarrow 0$ limit of the partition sum.

The partition function for the twisted (NS,NS) sectors is

$$
\begin{aligned}
& Z_{\mathrm{loc}}=\frac{V_{5} Z_{\Gamma}}{\left(2 \pi \sqrt{\alpha^{\prime} \tau_{2}}\right)^{5}} \sqrt{\frac{R^{2}}{\alpha^{\prime} \tau_{2}}} \sum_{\|s \gamma\| \neq 0, t \in \mathbf{Z}} e^{-\frac{\pi R^{2}}{\alpha^{\prime}} \frac{|t+s \tau|^{2}}{\tau_{2}}} \\
& \left|\frac{1}{2}\left(\frac{\vartheta\left[\begin{array}{l}
0 \\
0
\end{array}\right](0 \mid \tau)}{\eta^{3}}\right)^{3} \frac{\vartheta\left[\begin{array}{l}
s \gamma \\
t \gamma
\end{array}\right](0 \mid \tau)}{\vartheta\left[\begin{array}{c}
\frac{1}{2}+s \gamma \\
\frac{1}{2}+t \gamma
\end{array}\right](0 \mid \tau)}-e^{-\pi i t s \gamma} \frac{1}{2}\left(\frac{\vartheta\left[\begin{array}{c}
0 \\
1 / 2
\end{array}\right](0 \mid \tau)}{\eta^{3}}\right)^{3} \frac{\vartheta\left[\begin{array}{c}
s \gamma \\
1 / 2+t \gamma
\end{array}\right](0 \mid \tau)}{\vartheta\left[\begin{array}{c}
\frac{1}{2}+s \gamma \\
\frac{1}{2}+t \gamma
\end{array}\right](0 \mid \tau)}\right|^{2} .
\end{aligned}
$$

$Z_{\Gamma}$ is a Siegel-Narain theta function of signature $(5,5)$ corresponding to $T^{5}$. The behavior of the partition sum in the limit (1.6) does not depend on the details of the compactification.

To analyze the $\tau \rightarrow 0$ asymptotics of 2.3 we need the following asymptotics for $\tau=i \beta \rightarrow 0$, with $\beta$ real

$$
|\eta(\tau)| \rightarrow \frac{1}{\sqrt{\beta}} e^{-\frac{2 \pi}{24 \beta}}
$$

Similarly,

$$
\vartheta\left[\begin{array}{l}
\theta \\
\phi
\end{array}\right](0 \mid \tau) \rightarrow \begin{cases}\beta^{-1 / 2} e^{2 \pi i \theta} \tilde{q}^{\frac{1}{2}\|\phi\|^{2}}, & \text { if } \frac{1}{2}<\phi<1 \\
\beta^{-1 / 2}\left(1+e^{2 \pi i \theta}\right) \tilde{q}^{\frac{1}{2}\|\phi\|^{2}}, & \text { if } \phi=\frac{1}{2} \\
\beta^{-1 / 2} \tilde{q}^{\frac{1}{2}\|\phi\|^{2}}, & \text { if } 0 \leq \phi<\frac{1}{2}\end{cases}
$$

where $\tilde{q}=\exp (-2 \pi / \beta)$. Using these asymptotic formulae one can check that the leading behavior arises from $\epsilon_{1}=t=0$ in (2.3), (2.4). One finds that

$$
Z_{\mathrm{loc}} \sim \frac{1}{16} \sqrt{\frac{R^{2} \tau_{2}}{\alpha^{\prime}}} e^{\frac{2 \pi}{\tau_{2}}} \sum_{\|s \gamma\| \neq 0}^{\infty} e^{-\frac{\pi R^{2} \tau_{2}}{\alpha^{\prime}} s^{2}} \frac{1}{(\sin \pi s \gamma)^{2}}
$$

Before discussing the mathematical properties of (2.7) let us interpret the crucial factor $1 /(\sin \pi s \gamma)^{2}$ in (2.7). As mentioned in the discussion following (1.3), twisted sectors with $\|s \gamma\|<<1$ give rise to nearly delocalized states whose radial size scales like $1 /\|s \gamma\|$. This is reflected in the spectrum of $L_{0}$ in the following way. In the $s$-twisted sector, all states wind $s$ times around the $y$ circle, and thus have a large (for large $s$ ) ground state energy, 
of order $R s$ (or $L_{0} \sim(R s)^{2}$, see (1.3)). This gives the exponential prefactor in the sum (2.7). On top of this ground state energy, when $\|s \gamma\|$ is small, one finds a narrowly-spaced spectrum of states, associated with the twisted oscillators of the superfield $z$. This gives the inverse sine factor in (2.7). Thus, we see that this factor is directly related to the spatial extent of the twisted states.

If $\gamma$ is rational, $\gamma=p / q$ in lowest terms, (2.7) has a smooth $\tau_{2} \rightarrow 0$ limit. The limit is easily evaluated by setting $s=q \ell+j, 0 \leq j<q-1, \ell \in \mathbf{Z}$ to get

$$
Z_{\mathrm{loc}} \sim e^{2 \pi / \tau_{2}} \frac{1}{16} \sqrt{\frac{R^{2} \tau_{2}}{\alpha^{\prime}}} \sum_{j=1}^{q-1} \sum_{\ell \in \mathbf{Z}} e^{-\frac{\pi R^{2} q^{2} \tau_{2}}{\alpha^{\prime}}(\ell+j / q)^{2}} \frac{1}{(2 \sin \pi p j / q)^{2}} .
$$

Taking the $\tau_{2} \rightarrow 0$ limit we reproduce the familiar expression for the $\mathbf{C} / \mathbf{Z}_{q}$ orbifold [20]:

$$
\operatorname{dim} \mathcal{H}_{\text {loc }}(\gamma=p / q)=\frac{1}{16 q} \sum_{j=1}^{q-1} \frac{1}{(\sin \pi p j / q)^{2}}
$$

The trigonometric sum is easily evaluated [20],

$$
\operatorname{dim} \mathcal{H}_{\mathrm{loc}}(\gamma=p / q)=\frac{1}{48}\left(q-\frac{1}{q}\right)
$$

We see that for rational $\gamma$, the Melvin model is closely related to the corresponding $\mathbf{C} / \mathbf{Z}_{q}$ orbifold. Note that the result only depends on $q$ and hence is a highly erratic function of $\gamma \in \mathbf{Q}$. This is the first indication that we are dealing with delicate functions of $\gamma$.

\section{Comments on the sum in the case of irrational $\gamma$}

Now we turn to the case of $\gamma$ irrational. Stripping the universal exponential in (1.6) from (2.7), we see that to compute $g_{c l}$ we need to evaluate

$$
g(y ; \gamma):=\sqrt{y} \sum_{s \neq 0}^{\infty} e^{-\pi y s^{2}} \frac{1}{\sin ^{2} \pi s \gamma}
$$

in the limit $y \rightarrow 0$ (here $\left.y=\tau_{2} R^{2} / \alpha^{\prime}\right)$.

First, note that it is not obvious that the sum converges for finite $y$ (or $\tau_{2}$ ). Indeed, we will see in section 4 that for certain transcendental numbers it diverges. However, for

${ }^{4}$ Here $q$ is an integer, not to be confused with the modular parameter $q=e^{-2 \pi \tau_{2}}$. 
a "large" class of irrational numbers, including all algebraic numbers, it does converge. Recall the standard:

Definition: An irrational number is of Diophantine type $(K, \sigma)$ if for all $q \geq 1$,

$$
\sigma_{q}(\gamma):=\inf _{1 \leq s \leq q}\|s \gamma\| \geq \frac{K}{q^{1+\sigma}} .
$$

We denote the set of numbers of Diophantine type $(K, \sigma)$ by $\mathcal{D}(K, \sigma)$, and we also denote

$$
\mathcal{D}(\sigma):=\cup_{K>0} \mathcal{D}(K, \sigma) .
$$

If $\gamma$ is of Diophantine type $(K, \sigma)$ then $g(y ; \gamma)$ exists for all positive $y$. To show this we use

$$
2\|z\| \leq|\sin \pi z|<\pi\|z\|,
$$

(the best estimate valid for all real, non-integer $z$ ) to put upper and lower bounds on $g(y ; \gamma)$ :

$$
\sum_{s \neq 0} \frac{1}{\pi^{2}\|s \gamma\|^{2}} e^{-\pi y s^{2}}<\sum_{s \neq 0} e^{-\pi y s^{2}} \frac{1}{\sin ^{2} \pi s \gamma}<\sum_{s \neq 0} \frac{1}{4\|s \gamma\|^{2}} e^{-\pi y s^{2}} .
$$

If $\gamma$ is of type $(K, \sigma)$ then

$$
\frac{1}{\|s \gamma\|} \leq s^{1+\sigma} / K
$$

and hence by (3.5) the series is bounded above by a convergent sum.

Some interesting facts, which can be found in 12 16 are, first, that the set $\mathcal{D}(\sigma)$ is invariant under $S L(2, \mathbf{Z})$ (acting via fractional linear transformations on the elements of $\mathcal{D}(\sigma))$. Second, a theorem of Roth says that if $\gamma$ is algebraic of degree $\geq 2$ then it is of type $(K, \sigma)$ for all $\sigma>0$ and some $K$. 5

Diophantine approximation can give us some idea of what the asymptotics of $g(y ; \gamma)$ might be like. If there are many very good rational approximants to $\gamma$ then $\sin \pi s \gamma$ is "often" close to zero, and we expect a divergence as $y \rightarrow 0$. If good rational approximants to $\gamma$ are "rare" then the lower limit in (3.5) is more accurate and $g(y ; \gamma)$ will grow more slowly.

What we can say rigorously is that if $\gamma$ is of Diophantine type $(K, \sigma)$ then, from (3.6)

$$
C_{1} \leq g(y ; \gamma) \leq C_{2} y^{-\sigma-1}
$$

for some constants $C_{i}$. Therefore, we can define a non-negative number $b(\gamma)$ by :

$$
b(\gamma):=\inf \left\{b: \lim _{y \rightarrow 0} y^{b} g(y ; \gamma)=0\right\}
$$

We next show that $b(\gamma) \geq 1 / 2$.

5 A much easier theorem of Liouville, which is all we need to establish convergence for algebraic numbers, says that a degree $n \geq 2$ algebraic number is of Diophantine type $(K, n-2)$. 


\subsection{A lower bound for $b(\gamma)$}

To show that $g(y ; \gamma)$ always diverges for $y \rightarrow 0$ at least as strongly as $1 / \sqrt{y}$, we use the continued fraction expansion in positive integers $a_{n}$ :

$$
\gamma=\left[a_{0}, a_{1}, a_{2}, \ldots\right]=a_{0}+\frac{1}{a_{1}+\frac{1}{a_{2}+\cdots} .}
$$

The integers $a_{n}$ are known as partial quotients. The best rational approximants to $\gamma$ are always provided by the convergents

$$
\frac{p_{n}}{q_{n}}:=\left[a_{0}, \ldots, a_{n}\right]
$$

in the continued fraction expansion:

$$
\left|\gamma-\frac{p_{n}}{q_{n}}\right|<\frac{1}{q_{n}^{2}} .
$$

The $q_{n}$ grow exponentially as a function of $n$. Roughly speaking,

$$
q_{n} \sim c e^{\frac{1}{2} \lambda(\gamma) n}
$$

and more rigorously: 6

$$
\lambda(\gamma):=2 \lim _{n \rightarrow \infty} \frac{1}{n} \log q_{n} .
$$

The quantity $\lambda(\gamma)$ is known as the Lyapunov exponent of $\gamma$.

Taking a lower bound on $g(y ; \gamma)$ by summing only over $s=q_{n}$ and using (3.5), (3.11), one can show that

$$
g(y ; \gamma)>\frac{2}{\pi^{2}} \sqrt{y} \sum_{n=1}^{\infty} \frac{e^{-\pi y q_{n}^{2}}}{\left\|q_{n} \gamma\right\|^{2}}>\frac{2}{\pi^{2}} \sqrt{y} \sum_{n=1}^{\infty} q_{n}^{2} e^{-\pi y q_{n}^{2}} .
$$

Now, using (3.12) we see that the divergence as $y \rightarrow 0$ is at least as strong as

$$
g(y ; \gamma) \geq \frac{1}{y^{1 / 2}} \frac{2}{\pi^{3} \lambda(\gamma)} .
$$

3.2. $g(y ; \gamma)$ and the three gap theorem

Some further insight can be gained on the behavior of $g(y ; \gamma)$ as $y \rightarrow 0$ using the three-gap theorem of [26].

6 There are $\gamma$ 's for which the limit does not exist. 
The asymptotics of $g(y ; \gamma)$ as $y \rightarrow 0$ are the same as the $N \rightarrow \infty$ asymptotics of the sum

$$
g_{N}(\gamma)=N^{-1} \sum_{n=1}^{N}\|n \gamma\|^{-b}
$$

in the case $b=2$, where we identify $y \sim 1 / N^{2}$. It is useful in the discussion to keep $b$ general.

In the case $b \leq 0$, Kronecker's theorem, which tells us that $\|n \gamma\|$ are uniformly distributed implies

$$
g_{N}(\gamma) \rightarrow \int_{0}^{1}\|x\|^{-b} d x .
$$

The same holds when $0<b<1$, however one needs to assume $\gamma$ is Diophantine of type $\sigma$ where $\sigma$ depends on $b$. This is because values close to zero might cause some divergence since $\|x\|^{-b}$ is unbounded there. Estimates for the case $b=1$ are also classic [27].

We are here interested in $b>1$. In this case the sum is dominated by a finite number of terms. In order to see this order the points $\|n \gamma\|(n=1, \ldots, N)$ in the interval $[0,1 / 2]$ and label them by

$$
0<\xi_{1}<\ldots<\xi_{N}<1 / 2
$$

So

$$
g_{N}(\gamma)=N^{-1} \sum_{n=1}^{N} \xi_{n}^{-b} .
$$

We now summarize the results of [26]. Label the fractional parts of $n \gamma$ by

$$
0<\eta_{1}<\ldots<\eta_{N}<1
$$

The "three gap theorem" states that every spacing $\eta_{n+1}-\eta_{n}$ is equal to either $\alpha, \beta$ or $\alpha+\beta$, where $\alpha=\eta_{1}$ and $\beta=1-\eta_{N}$. In [26] one finds formulae for $\alpha, \beta$ in terms of the continued fraction approximation of $\gamma$. In particular $\alpha$ and $\beta$ in general have no asymptotics as $N \rightarrow \infty$.

Now if $\gamma$ is of bounded type (i.e. if the partial quotients $a_{n}$ are bounded by some constant), one finds immediately from the three gap theorem that there are constants $c, C>0$ such that

$$
c / N \leq \alpha \leq C / N, \quad c / N \leq \beta \leq C / N .
$$

It is thus natural to write

$$
g_{N}(\gamma)=N^{b-1} \sum_{n=1}^{N}\left(N \xi_{n}\right)^{-b}
$$


Since the gaps between the $N \eta_{n}$ are bounded from below by a constant, we have $c^{\prime} n \leq$ $N \xi_{n} \leq C^{\prime} n$ for suitable constants $c^{\prime}, C^{\prime}>0$. Therefore (and provided $b>1$ ), given any error threshold $\epsilon>0$ we find an $M_{\epsilon}$ so that

$$
\limsup _{N \rightarrow \infty} \sum_{n=M_{\epsilon}}^{N}\left(N \xi_{n}\right)^{-b}<\epsilon .
$$

Hence

$$
g_{N}(\gamma)=N^{b-1} \sum_{n=1}^{M_{\epsilon}-1}\left(N \xi_{n}\right)^{-b}+O\left(\epsilon N^{b-1}\right) .
$$

This means $g_{N}(\gamma)$ is of order $N^{b-1}$, and furthermore arbitrarily well approximable by a finite number of terms. Recall the $N \xi_{n}, n=1, \ldots, M_{\epsilon}$, are bounded from above and below and have an explicit expression in terms of the continued fraction approximants of $\gamma$.

One important consequence of these considerations is that $\sqrt{y} g(y ; \gamma)$ has no good asymptotics. The value remainds bounded but fluctuates as $y \rightarrow 0$. Nevertheless, as we will see in the next section, this value is governed by a definite probability law.

\subsection{The regularized dimension is a random variable in a probability distribution}

We have seen in the previous subsection that the asymptotic $y \rightarrow 0$ behaviour of the function

$$
g(y ; \gamma)=\sqrt{y} \sum_{m \in \mathbf{Z}-\{0\}} \frac{e^{-\pi m^{2} y}}{\sin ^{2}(\pi m \gamma)}
$$

is determined by the continued fraction expansion of $\gamma$. We will here refine our analysis by exploiting the dynamical properties of the geodesic flow on the modular surface. The connection between continued fraction dynamics and geodesic flow is non-trivial but well understood, cf. [28,29].

To explain the strategy, note that

$$
\tilde{g}(y ; \gamma)=\sqrt{y} \sum_{(m, n) \in \mathbf{Z}^{2}-\{\overrightarrow{0}\}} \frac{e^{-\pi y m^{2}}}{\pi^{2}(m \gamma+n)^{2}}
$$

has the same asymptotic behaviour as $g(y ; \gamma)$, up to an error of order $O(1)$, i.e.,

$$
g(y ; \gamma)=\tilde{g}(y ; \gamma)+O(1)
$$


uniformly for all $\gamma$. (To prove this use the identity

$$
\frac{1}{\sin ^{2}(\pi m \gamma)}=\frac{1}{\pi^{2}} \sum_{n=-\infty}^{+\infty} \frac{1}{(m \gamma+n)^{2}}
$$

and add and subtract the $(m=0, n \neq 0)$ terms by hand.) The main idea is now to construct a certain modular function $F(M)$ on $S L(2, \mathbf{Z}) \backslash S L(2, \mathbf{R})$, such that

$$
\sqrt{y} \tilde{g}(y ; \gamma)=F(M(t)), \quad t=-\log y \rightarrow \infty
$$

where $M(t) \in S L(2, \mathbf{R})$ is evaluated along the geodesic

$$
M(t)=\left(\begin{array}{ll}
1 & \gamma \\
0 & 1
\end{array}\right)\left(\begin{array}{cc}
e^{-t / 2} & 0 \\
0 & e^{t / 2}
\end{array}\right), \quad t \geq 0 .
$$

The asymptotics of $\sqrt{y} \tilde{g}(y ; \gamma)$ is now entirely determined by the geometric distribution of the geodesic associated with a particular value of $\gamma$. For example:

(a) If $\gamma$ is a quadratic irrational, then the geodesic $M(t)$ is asymptotic to a closed geodesic with period $T_{\gamma}$. Hence

$$
e^{-t / 2} \tilde{g}\left(e^{-t} ; \gamma\right) \sim \phi(t)
$$

where $\phi(t)$ is a bounded periodic function with period $T_{\gamma}$.

(b) If $\gamma$ is badly approximable by rationals (i.e., Diophantine of bounded type), then the geodesic $M(t)$ is asymptotic to a geodesic which never leaves a bounded set in $S L(2, \mathbf{Z}) \backslash S L(2, \mathbf{R})$. Hence $e^{-t / 2} \tilde{g}\left(e^{-t} ; \gamma\right)$ is bounded for all $t$. Our analysis will show that in general $F(M)$ is a non-constant function, hence $e^{-t / 2} \tilde{g}\left(e^{-t} ; \gamma\right)$ does not converge to a constant.

(c) For almost all $\gamma$ (with respect to Lebesgue measure) the corresponding geodesic $M(t)$ becomes equidistributed in $S L(2, \mathbf{Z}) \backslash S L(2, \mathbf{R})$, a consequence of the ergodicity of the geodesic flow. Hence the fluctuations of $e^{-t / 2} \tilde{g}\left(e^{-t} ; \gamma\right)$ on some long stretch $[0, T]$ $(T \rightarrow \infty)$ have the same probability distribution as the function $F(M)$, where $M$ varies over $S L(2, \mathbf{Z}) \backslash S L(2, \mathbf{R})$. That is,

$$
\frac{1}{T} \int_{0}^{T} \delta\left(X-e^{-t / 2} g\left(e^{-t} ; \gamma\right)\right) d t \longrightarrow P(X)=\int_{S L(2, \mathbf{Z}) \backslash S L(2, \mathbf{R})} \delta(X-F(M)) d M .
$$

Interestingly, the limit distribution has an algebraic tail, $P(X) \sim A X^{-3 / 2}$, and hence no first moment. See Theorem 1 in the appendix for details. 


\section{Divergence for finite $\tau$}

In the previous section we discussed the behavior of the torus partition sum (2.4) in the limit $\tau \rightarrow 0$. In this section we will see that for some $\gamma$, the sum over twisted sectors (and thus (3.1)) diverge for finite $\tau$. This point has been mentioned briefly in 30].

The dangerous factor in the partition sum (2.4) is the function

$$
\vartheta\left[\begin{array}{c}
\frac{1}{2}+s \gamma \\
\frac{1}{2}
\end{array}\right](0 \mid \tau)
$$

which appears in the denominator; it becomes very small when $\|s \gamma\|<<1$. As we have seen, this is due to the fact that the corresponding states are nearly delocalized.

Consider

$$
F(x):=\vartheta\left[\begin{array}{c}
\frac{1}{2}+x \\
\frac{1}{2}
\end{array}\right](0 \mid \tau) .
$$

It is easy to check that $F(x+1)=F(x)$ and $e^{-i \pi x} F(x)$ is an odd function of $x$ given at small $x$ by

$$
e^{-i \pi x} F(x)=-\left(2 \pi \tau \eta^{3}\right) x++\left(2 \pi^{3} \tau^{3} E_{2}-12 \pi^{2} i \tau^{2}\right) \eta^{3} \frac{x^{3}}{3 !}+\cdots
$$

The convergence of the sum over twisted sectors of (2.4) for fixed $\tau$ is controlled by

$$
\sum_{s=1}^{\infty} \frac{1}{\|s \gamma\|^{2}} e^{-\frac{\pi R^{2}}{\alpha^{\prime}} s^{2} \frac{|\tau|^{2}}{\tau_{2}}}
$$

As discussed in the previous section, for $\gamma$ of Diophantine type $(K, \sigma)$ the sum (4.4) converges. On the other hand, for certain Liouville numbers the sum actually diverges. To show this, consider the subsum given by $s=q_{n}$ where $q_{n}$ is the denominator of the convergents of $\gamma,(3.10)$. Then [15]:

$$
\frac{1}{q_{n}+q_{n+1}}<\left\|q_{n} \gamma\right\|<\frac{1}{q_{n+1}} .
$$

Thus, (4.4) is bounded from below by

$$
\sum_{n} q_{n+1}^{2} e^{-\kappa q_{n}^{2}}
$$

where $\kappa$ is some constant. Now if

$$
2 \log q_{n+1}-\kappa q_{n}^{2}=\mathcal{O}(1)
$$


or is even bounded below by $-\log n$ then the series (4.6) diverges.

We can thus construct numbers for which the series (4.6) diverges by considering $\gamma$ of the form

$$
\gamma=\sum_{n=1}^{\infty} \frac{1}{10^{f(n)}}
$$

for certain rapidly increasing functions $f(n)$. Indeed we may take the subsum with $s=$ $10^{f(n)}$. Then

$$
\left|\gamma-\sum_{j=1}^{n} \frac{1}{10^{f(j)}}\right|<\frac{2}{10^{f(n+1)}} .
$$

Now consider any function $f(n)$ that satisfies an equation of the form

$$
f(n+1)=f(n)+\kappa 10^{2 f(n)}+g(n)
$$

where $g(n)$ is, say, any positive function of $n$. Then, using $q_{n}=10^{f(n)}$ and $q_{n+1}^{2}<\left\|q_{n} \gamma\right\|^{-2}$ we see that for such functions $f(n)$ the series (4.4) diverges. Thus, there are continuum many transcendental numbers for which the sum diverges.

\section{An alternative regularization using continued fractions}

In the previous sections we discussed the regularized number of localized states given by the partition sum (1.4) in the limit $\tau \rightarrow 0$. We saw that there are some irrational numbers for which the sum over localized states diverges even for finite $\tau$. This divergence is due to the effect of "nearly untwisted strings" with $\|w \gamma\|$ small. It is natural to ask whether one can regularize this divergence in some other way, consistent with conformal symmetry and modular invariance.

Replacing $\mathbf{C}$ by, say, a sphere of finite radius breaks conformal symmetry, and introduces subtle questions of orders of limits. Similarly, putting a cutoff on the sum over twist sectors breaks modular invariance. One simple way to regulate the volume divergence is to use the continued fraction expansion of $\gamma$

$$
\gamma=\left[0, a_{1}, a_{2}, \ldots\right]
$$

Cutting off the continued fraction at a finite place leads to the rational convergents:

$$
\gamma^{(n)}:=\left[0, a_{1}, a_{2}, \ldots, a_{n}\right]:=\frac{p_{n}}{q_{n}}
$$


For the rational twists $\gamma^{(n)}$ we have a clear separation of localized from delocalized states and the regularized dimension of the space of localized states is (2.10)

$$
\operatorname{dim} \mathcal{H}_{\mathrm{loc}}\left(\gamma^{(n)}\right)=\frac{1}{48}\left(q_{n}-\frac{1}{q_{n}}\right)
$$

Similarly, other correlation functions in the orbifold CFT are well-defined for finite $n$.

One can formally think of the original orbifold with twist parameter $\gamma$ as the limit $n \rightarrow \infty$ of (5.2). Of course, $q_{n} \rightarrow \infty$ as $n \rightarrow \infty$, but it does so at different rates for different $\gamma$ 's; the rate depends sensitively on $\gamma$ through the Lyapunov exponent (3.13).

The exponential growth of $q_{n}$ suggests that we should define an "entropy of delocalization" by considering the limiting behavior of $S_{n}(\gamma)=\log \operatorname{dim} \mathcal{H}_{\text {loc }}\left(\gamma^{(n)}\right)$. With this measure of delocalization we have

$$
\frac{\log \operatorname{dim} \mathcal{H}_{\mathrm{loc}}\left(\gamma_{1}\right)}{\log \operatorname{dim} \mathcal{H}_{\mathrm{loc}}\left(\gamma_{2}\right)}=\lim _{n \rightarrow \infty} \frac{S_{n}\left(\gamma_{1}\right)}{S_{n}\left(\gamma_{2}\right)}=\frac{\lambda\left(\gamma_{1}\right)}{\lambda\left(\gamma_{2}\right)}
$$

Some interesting facts about $\lambda(\gamma)$, which can be found in [31], are the following. First, for almost every $\gamma, \lambda(\gamma)$ is given by Khinchin's constant

$$
\lambda_{0}=\frac{\pi^{2}}{6 \log 2}
$$

Moreover, the range of $\lambda(\gamma)$ as $\gamma$ runs over irrational numbers in $(0,1)$ is

$$
\left[2 \log \frac{1+\sqrt{5}}{2}, \infty\right)
$$

Thus, the entropy of delocalization is a nontrivial function of the twist parameter $\gamma$ of the Melvin model.

\section{Remarks}

1. One very interesting property of the Lyapunov exponent $\lambda(\gamma)$ is that it is invariant under $S L(2, \mathbf{Z})$ acting on $\gamma$ via fractional linear transformations. This is easily seen since $\gamma \rightarrow \gamma+1$ obviously does not change the exponent while, for $\gamma=\left[0, a_{1}, a_{2}, \ldots\right]$ we have $1 / \gamma=\left[a_{1}, a_{2}, a_{3}, \ldots\right]$, so $\{1 / \gamma\}=\left[0, a_{2}, a_{3}, \ldots\right]$.

2. The Lyapunov exponent of $\gamma$ is indeed a Lyapunov exponent for a dynamical system, namely that defined by the Gauss map $T(x)=\{1 / x\}$, which shifts the entries of the continued fraction expansion. In this context it is quite amusing to note that a naive analysis of the GLSM description for the Melvin model discussed in [11] appears to 
lead to a connection between 2D RG flow and the Gauss map. If we choose a GLSM with gauge group $\mathbf{R}$ with gauge group action $\left(X_{1}, X_{2}, P\right) \rightarrow\left(e^{i \gamma \theta} X_{1}, e^{-i \theta} X_{2}, P+i \theta\right)$ then the standard analysis of the $D$-term equation

$$
\gamma\left|X_{1}\right|^{2}-\left|X_{2}\right|^{2}=p_{1}
$$

suggests that the Melvin geometry with twist parameter $\gamma$ and radius $R$ flows to that with twist parameter $\{1 / \gamma\}$ and radius $R / \gamma$. Thus, at least as long as $R$ remains small, the flow from the UV to the IR acts as a Gauss map on $\gamma$.

3. Some other interesting relations of Lyapunov exponents to areas of physics are explored in 32.

4. The approach of this section has the advantage that it can be easily extended to other twisted tori geometries $\mathbf{C}^{d} \times \mathbf{R}^{d^{\prime}} / \Gamma$, where $\Gamma$ acts by linear transformations in $\mathbf{C}^{d}$ and by translations in $\mathbf{R}^{d^{\prime}}$.

\section{Melvin models in string theory}

So far we have been focusing on the conformal field theory of the Melvin orbifold, and the divergences associated with the sums over twisted sectors in defining partition functions in this CFT. In string theory we have the further complication that we must integrate amplitudes over moduli space.

We have seen that for certain irrational numbers $\gamma$, the partition function of the twisted (NS,NS) sectors, which contain spacetime bosons, is divergent for fixed $\tau$. In type II string theory, what enters into the torus amplitude - the one loop contribution to the cosmological constant - is the difference of spacetime bosons and fermions,

$$
\sqrt{\frac{R^{2}}{\alpha^{\prime} \tau_{2}}} \sum_{(s, t) \neq(0,0)} e^{-\frac{\pi R^{2}}{\alpha^{\prime}} \frac{|t+s \tau|^{2}}{\tau_{2}}}\left|\frac{\sum_{\epsilon_{1}, \epsilon_{2}} \eta_{\epsilon_{1}, \epsilon_{2}}\left(\frac{\vartheta\left[\begin{array}{c}
\epsilon_{2} \\
\epsilon_{1}
\end{array}\right](0 \mid \tau)}{\eta}\right)^{3} \vartheta\left[\begin{array}{c}
\epsilon_{2}+s \gamma \\
\epsilon_{1}+t \gamma
\end{array}\right](0 \mid \tau)}{\vartheta\left[\begin{array}{l}
\frac{1}{2}+s \gamma \\
\frac{1}{2}+t \gamma
\end{array}\right](0 \mid \tau)}\right|^{2}
$$

where $\eta_{\epsilon_{1} \epsilon_{2}}= \pm 1$ are described in [25]. It is still the case that the denominator of (6.1) is small in sectors with $t=0$ and small $\|s \gamma\|$ (4.1). However, the numerator goes to zero as well, due to the standard Riemann identity, or more physically because when $\|s \gamma\| \rightarrow 0$, one is approaching the untwisted contribution, which vanishes due to the standard supersymmetric cancellations. However, when $t \gamma$ is a good approximation to 
an odd integer the numerator does not cancel the denominator. 7 In this case there are potential divergences such as those discussed in section 4 .

In addition to this, for other one loop amplitudes we expect that the sum over twisted sectors $s$ will again be problematic. This is compounded by the fact that we must integrate over moduli space, since the integral over $\tau_{2}$ has the form

$$
\int^{\infty} d \tau_{2} \tau_{2}^{\nu} e^{-s^{2} \tau_{2}} \sim \frac{1}{s^{(\nu+1) / 2}}
$$

Thus the exponential suppression in $s$ is replaced by power law suppression, which can be easily overwhelmed by $1 /\|s \gamma\|^{2}$ even for algebraic irrationals $\gamma$. For this reason, it is far from clear to us that Melvin spacetimes with irrational values of $\gamma$ are well-defined string backgrounds. This issue merits further investigation.

\section{Discussion}

The discussion of the previous sections suggests that physics is not continuous as a function of $\gamma$. This may seem physically unreasonable. How can continuous changes of a magnetic field lead to discontinuous conformal field theory or string theory amplitudes? There is a well-known precedent for this kind of behavior, namely the Azbel-Hofstadter model of an electron in a magnetic field in the presence of a periodic potential [33]. The spectrum of the Schrödinger operator is a sensitive function of the magnetic field, and depends on its arithmetic nature.

It has been claimed that Melvin models provide a smooth interpolation between IIB and $0 \mathrm{~A}$ string theory and this has been used to argue that the endpoint of $0 \mathrm{~A}$ tachyon condensation is the IIB theory [34, 35, 11]. It is possible that the interpolating string theories with irrational $\gamma$ do not exist, thus calling these claims into question.

A natural class of questions which arise in the context of our considerations have to do with asking "how many" $\gamma$ 's or "how often" a $\gamma$ leads to a divergent model, or to a model with fixed Lyapunov exponent, and so forth. These are subtle and difficult questions. They have been the subject of much research. To quote one nice result [31], the set of $\gamma$ such that $\lambda(\gamma)$ takes a value larger than the Khinchin constant $\lambda_{0}(5.5)$ has positive Hausdorff dimension. Further discussion of such matters would take us into the subject of measure and category [36], so this seems a good place to stop.

7 We thank the referee for pointing out this subtlety. 
Acknowledgements: We would like to thank J. Harvey and E. Martinec for collaboration on related matters and for useful discussions. We would like to thank J. Lagarias, J. Maldacena, and B. Pioline for useful discussions and correspondence. We also thank the LPTHE at Jussieu, Paris, for hospitality while this paper was written. The work of GM is supported in part by DOE grant DE-FG02-96ER40949. That of DK is supported in part by DOE grant DE-FG02-90ER40560. JM was supported by an EPSRC Advanced Research Fellowship and the EC Research Training Network (Mathematical Aspects of Quantum Chaos) HPRN-CT-2000-00103.

\section{Appendix A. Detailed proof of (3.31)}

\section{A.1. Sums over lattice points}

For any $M \in S L(2, \mathbf{R})$ consider the sum

$$
F(M)=\sum_{\vec{m} \in \mathbf{Z}^{2}-\{\overrightarrow{0}\}} f(\vec{m} M)
$$

where $\vec{m}$ runs over all non-zero integer row vectors, and $f$ is a positive function on $\mathbf{R}^{2}$. Since the modular group $S L(2, \mathbf{Z})$ leaves the lattice $\mathbf{Z}^{2}$ and the origin $\overrightarrow{0}$ invariant, we have immediately

$$
F(K M)=F(M)
$$

for every $K \in S L(2, \mathbf{Z})$. $F$ may thus be viewed as a function on the homogeneous space $S L(2, \mathbf{Z}) \backslash S L(2, \mathbf{R})$. There is a simple formula for the average of $F$ with respect to Haar measure $d M$, normalized as a probability measure so that

$$
\int_{S L(2, \mathbf{Z}) \backslash S L(2, \mathbf{R})} d M=1 .
$$

We then have

$$
\int_{S L(2, \mathbf{Z}) \backslash S L(2, \mathbf{R})} F(M) d M=\int_{\mathbf{R}^{2}} f(\vec{x}) d x .
$$

This is a special case of Siegel's weight formula for $S L(d, \mathbf{R})$; for a proof see e.g. Theorem 3.15 of [37].

The function $\tilde{g}(y ; \gamma)$ is connected to an automorphic function $F$ of the above form: choose

$$
f\left(x_{1}, x_{2}\right)=\frac{e^{-\pi x_{1}^{2}}}{\pi^{2} x_{2}^{2}} .
$$


Then, at the point

$$
M=\left(\begin{array}{ll}
1 & x \\
0 & 1
\end{array}\right)\left(\begin{array}{cc}
y^{1 / 2} & 0 \\
0 & y^{-1 / 2}
\end{array}\right)
$$

we have

$$
F(M)=\sqrt{y} \tilde{g}(y ; x)
$$

The space $S L(2, \mathbf{Z}) \backslash S L(2, \mathbf{R})$ can be identified with the unit tangent bundle of the modular surface by means of the Iwasawa decomposition

$$
M=\left(\begin{array}{cc}
1 & x \\
0 & 1
\end{array}\right)\left(\begin{array}{cc}
y^{1 / 2} & 0 \\
0 & y^{-1 / 2}
\end{array}\right)\left(\begin{array}{cc}
\cos (\theta / 2) & \sin (\theta / 2) \\
-\sin (\theta / 2) & \cos (\theta / 2)
\end{array}\right)
$$

$z=x+i y$ are the standard upper half plane coordinates and the angle $\theta \in[0,2 \pi)$ describes the direction of the unit tangent vector at $z$. This identification induces the following action of a matrix $\left(\begin{array}{ll}a & b \\ c & d\end{array}\right) \in S L(2, \mathbf{R})$ on a point $M=(z, \theta)$ :

$$
\left(\begin{array}{ll}
a & b \\
c & d
\end{array}\right)(z, \theta)=\left(\frac{a z+b}{c z+d}, \theta-2 \arg (c z+d)\right) .
$$

A fundamental domain of $S L(2, \mathbf{Z})$ in these coordinates is

$$
\mathcal{F}=\{(z, \theta):|z|>1,|x|<1 / 2, \theta \in[0,2 \pi)\}
$$

The normalized Haar measure reads

$$
d M=\frac{3}{2 \pi^{2}} \frac{d x d y d \theta}{y^{2}}
$$

The geodesic flow on $S L(2, \mathbf{Z}) \backslash S L(2, \mathbf{R})$ is represented by the right translation

$$
M(0) \mapsto M(t)=M(0) \Phi^{t}, \quad \Phi^{t}=\left(\begin{array}{cc}
e^{-t / 2} & 0 \\
0 & e^{t / 2}
\end{array}\right)
$$

The values of $\tilde{g}(y ; x)$ are thus those of $F(M)$ evaluated along a geodesic $M=M(t)$ with initial condition $M(0)=\left(\begin{array}{ll}1 & x \\ 0 & 1\end{array}\right)$.

\section{A.2. Singularities}

To analyze the singularities of $F(M)$ we split

$$
F(M)=F_{0}(M)+F_{1}(M)
$$


where $F_{0}, F_{1}$ are defined in the same way as $F$ above with $f$ replaced with

$$
f_{0}\left(x_{1}, x_{2}\right)=\frac{e^{-\pi x_{1}^{2}}}{\pi^{2} x_{2}^{2}} \chi_{0}\left(x_{2}\right)
$$

and

$$
f_{1}\left(x_{1}, x_{2}\right)=\frac{e^{-\pi x_{1}^{2}}}{\pi^{2} x_{2}^{2}} \chi_{1}\left(x_{2}\right),
$$

respectively. $\chi_{0}$ and $\chi_{1}$ are continuous functions with values in $[0,1]$ such that $\chi_{0}(x)+$ $\chi_{1}(x)=1$, and

$$
\chi_{1}(x)= \begin{cases}1, & x \in[-\epsilon, \epsilon] \\ 0, & x \notin[-\epsilon(1+\epsilon), \epsilon(1+\epsilon)]\end{cases}
$$

for some fixed $\epsilon>0$. (The extra $(1+\epsilon)$ factor is used to accommodate the continuity of $\chi_{1}$; we think of $\chi_{0}$ and $\chi_{1}$ as smoothed characteristic functions.)

By construction, $F_{0}$ is a continuous function on all of $S L(2, \mathbf{Z}) \backslash S L(2, \mathbf{R})$. This manifold has one cusp at $y \rightarrow \infty$. The asymptotic behaviour is here

$$
\begin{aligned}
F_{0}(M) & \sim \sum_{n \neq 0} f_{0}\left(\left(0, n y^{-1 / 2}\right)\left(\begin{array}{cc}
\cos (\theta / 2) & \sin (\theta / 2) \\
-\sin (\theta / 2) & \cos (\theta / 2)
\end{array}\right)\right) \\
& \sim C_{0}(\theta) y^{1 / 2}
\end{aligned}
$$

as $y \rightarrow \infty$, where

$$
\begin{aligned}
C_{0}(\theta) & =\int_{-\infty}^{\infty} f_{0}\left((0, r)\left(\begin{array}{cc}
\cos (\theta / 2) & \sin (\theta / 2) \\
-\sin (\theta / 2) & \cos (\theta / 2)
\end{array}\right)\right) d r \\
& =\frac{1}{\pi^{2}} \int_{-\infty}^{\infty} \frac{e^{-\pi[r \sin (\theta / 2)]^{2}}}{[r \cos (\theta / 2)]^{2}} \chi_{0}(r \cos (\theta / 2)) d r .
\end{aligned}
$$

Note that $C_{0}(\theta)=O(1)$ for all $\theta$.

We re-write $F_{1}$ as a sum over primitive lattice points $\vec{p}$,

$$
F_{1}(M)=\sum_{l=1}^{\infty} \sum_{\vec{p}} f_{1}(l \vec{p} M)
$$

For every primitive lattice point $\vec{p}$ there is a $K \in S L(2, \mathbf{Z})$ such that $\vec{p}=(0,1) K$. The subgroup $\Gamma_{\infty} \subset S L(2, \mathbf{Z})$ of elements $K$ such that $(0,1) K=(0,1)$ is

$$
\Gamma_{\infty}=\left\{\left(\begin{array}{cc}
1 & n \\
0 & 1
\end{array}\right): n \in \mathbf{Z}\right\}
$$


and hence there is a one-to-one correspondence between primitive lattice points and the coset $\Gamma_{\infty} \backslash S L(2, \mathbf{Z})$. We have therefore

$$
F_{1}(M)=\sum_{l=1}^{\infty} \sum_{K \in \Gamma_{\infty} \backslash S L(2, \mathbf{Z})} f_{1}((0, l) K M)
$$

Due to the rapid decay of $f_{1}$ this is essentially a finite sum. To understand the singularities of $F_{1}$ consider the term corresponding to $K=1$,

$$
\sum_{l=1}^{\infty} f_{1}((0, l) M)=\frac{y}{\cos (\theta / 2)^{2}} \sum_{l=1}^{\infty} \frac{e^{-\pi l^{2} y^{-1} \sin (\theta / 2)^{2}}}{\pi^{2} l^{2}} \chi_{1}\left(l y^{-1 / 2} \cos (\theta / 2)\right)
$$

The main singularity of this function is at $\theta=\pi$, and we note that for $\theta \rightarrow \pi$

$$
\sum_{l=1}^{\infty} f_{1}((0, l) M) \sim \frac{4 y}{\pi^{2}(\theta-\pi)^{2}} \sum_{l=1}^{\infty} \frac{e^{-\pi l^{2} y^{-1}}}{l^{2}}
$$

The singularities of $F_{1}(M)$ are the images of the two-dimensional subspace $\{(z, \theta): \theta=\pi\}$ under the action of $\left(\begin{array}{ll}a & b \\ c & d\end{array}\right) \in \Gamma_{\infty} \backslash S L(2, \mathbf{Z})$,

$$
\left(\begin{array}{ll}
a & b \\
c & d
\end{array}\right)\{(z, \theta): \theta=\pi\}=\{(z, \theta): \theta=\pi-2 \arg (c z+d)\}
$$

where $(c, d)$ runs over all primitive lattice points in $\mathbf{Z}^{2}-\{\overrightarrow{0}\}$.

\section{A.3. Limit theorems}

Our main application of the above construction is the following.

Theorem 1. There is a probability density $P(X)$ on $\mathbf{R}_{+}$with the following properties:

1. There is a set of $x$ of full measure such that, for any bounded continuous function $\phi: \mathbf{R}_{+} \rightarrow \mathbf{R}$,

$$
\lim _{T \rightarrow \infty} \frac{1}{T} \int_{0}^{T} \phi\left(e^{-t / 2} g\left(e^{-t} ; x\right)\right) d t=\int_{0}^{\infty} \phi(X) P(X) d X
$$

2. For any bounded continuous function $\phi: \mathbf{R}_{+} \rightarrow \mathbf{R}$,

$$
\lim _{y \rightarrow 0} \int_{0}^{1} \phi(\sqrt{y} g(y ; x)) d x=\int_{0}^{\infty} \phi(X) P(X) d X
$$


3. As $X \rightarrow \infty$,

$$
P(X) \sim A X^{-3 / 2},
$$

with

$$
A=\frac{3}{2 \pi^{3}} \int_{0}^{\infty}\left(\sum_{l=1}^{\infty} \frac{e^{-\pi l^{2} y^{-1}}}{l^{2}}\right)^{1 / 2} \frac{d y}{y^{3 / 2}} .
$$

Note that the limiting distribution does not possess a first moment,

$$
\int_{0}^{\infty} X P(X) d X=\infty
$$

Thus, there is no "average" value of $g_{c l}$ for Melvin models.

To prove the above limit theorem, we note that the ergodicity of the geodesic flow and the equidistribution of long closed horocycles on $S L(2, \mathbf{Z}) \backslash S L(2, \mathbf{R})$ imply the following statements, cf. [37].

\section{Theorem 2}

1. There is a set of $x$ of full measure such that, for any bounded continuous function $G: S L(2, \mathbf{Z}) \backslash S L(2, \mathbf{R}) \rightarrow \mathbf{C}$,

$$
\lim _{T \rightarrow \infty} \frac{1}{T} \int_{0}^{T} G\left(\left(\begin{array}{cc}
1 & x \\
0 & 1
\end{array}\right) \Phi^{t}\right) d t=\int_{S L(2, \mathbf{Z}) \backslash S L(2, \mathbf{R})} G(M) d M ;
$$

2. For any bounded continuous function $G: S L(2, \mathbf{Z}) \backslash S L(2, \mathbf{R}) \rightarrow \mathbf{C}$,

$$
\lim _{y \rightarrow 0} \int_{0}^{1} G\left(\left(\begin{array}{ll}
1 & x \\
0 & 1
\end{array}\right)\left(\begin{array}{cc}
y^{1 / 2} & 0 \\
0 & y^{-1 / 2}
\end{array}\right)\right) d x=\int_{S L(2, \mathbf{Z}) \backslash S L(2, \mathbf{R})} G(M) d M .
$$

Now take any compactly supported continuous function $\phi: \mathbf{R}_{+} \rightarrow \mathbf{R}$ and set $G(M)=$ $\phi(F(M))$. Then for $\epsilon>0$ small enough

$$
G(M)=\phi\left(F_{0}(M)\right)
$$

and hence $G(M)$ is bounded continuous, in view of the above singularity analysis. Theorem 2 therefore implies the first two statements of Theorem 1, for compactly supported continuous test functions $\phi$, with

$$
P(X)=\int_{S L(2, \mathbf{Z}) \backslash S L(2, \mathbf{R})} \delta(X-F(M)) d M .
$$

The extension to bounded continuous $\phi$ follows from a standard probabilistic argument. 


\section{A.4. Tail estimates}

Consider first the large $X$ asymptotics of

$$
\begin{aligned}
P_{1}(X) & =\int_{S L(2, \mathbf{Z}) \backslash S L(2, \mathbf{R})} \delta\left(X-F_{1}(M)\right) d M \\
& =\int_{S L(2, \mathbf{Z}) \backslash S L(2, \mathbf{R})} \delta\left(X-\sum_{K \in \Gamma_{\infty} \backslash S L(2, \mathbf{Z})} \sum_{l=1}^{\infty} f_{1}((0, l) K M)\right) d M \\
& \sim \int_{S L(2, \mathbf{Z}) \backslash U_{\sigma}} \delta\left(X-\sum_{K \in \Gamma_{\infty} \backslash S L(2, \mathbf{Z})} \sum_{l=1}^{\infty} f_{1}((0, l) K M)\right) d M,
\end{aligned}
$$

where $U_{\sigma}=S L(2, \mathbf{Z})\{(z, \theta): \theta \in \pi+[-\sigma, \sigma]\}$ is a small neighbourhood of the singular set (A.24) on which $F_{1}(M)$ is large. Since the $K$ sum is essentially finite, we may choose $\sigma>0$ small enough so that the overlap of the neighbourhoods $K\{(z, \theta): \theta \in \pi+[-\sigma, \sigma]\}$ for different $K$ is negligible. Hence for $X \rightarrow \infty$,

$$
\begin{aligned}
P_{1}(X) & \sim \sum_{K \in \Gamma_{\infty} \backslash S L(2, \mathbf{Z})} \int_{S L(2, \mathbf{Z}) \backslash U_{\sigma}} \delta\left(X-\sum_{l=1}^{\infty} f_{1}((0, l) K M)\right) d M \\
& =\int_{\Gamma_{\infty} \backslash U_{\sigma}} \delta\left(X-\sum_{l=1}^{\infty} f_{1}((0, l) M)\right) d M \\
& =\frac{3}{2 \pi^{2}} \int_{0}^{1} \int_{0}^{\infty} \int_{\pi-\sigma}^{\pi+\sigma} \delta\left(X-\sum_{l=1}^{\infty} f_{1}((0, l) M)\right) \frac{d x d y d \theta}{y^{2}} \\
& \sim \frac{3}{2 \pi^{2}} \int_{0}^{\infty} \int_{-\sigma}^{\sigma} \delta\left(X-\frac{4 h(y)}{\theta^{2}}\right) \frac{d y d \theta}{y^{2}} \\
& =\frac{3}{2 \pi^{2} X^{3 / 2}} \int_{0}^{\infty} \sqrt{h(y)} \frac{d y}{y^{2}}
\end{aligned}
$$

where

$$
h(y)=\frac{y}{\pi^{2}} \sum_{l=1}^{\infty} \frac{e^{-\pi l^{2} y^{-1}}}{l^{2}} .
$$

Since $F_{0}(M)$ has its only singularity in the cusp $y \rightarrow \infty$,

$$
\begin{aligned}
P_{0}(X) & =\int_{S L(2, \mathbf{Z}) \backslash S L(2, \mathbf{R})} \delta\left(X-F_{0}(M)\right) d M \\
& \sim \frac{3}{2 \pi^{2}} \int_{0}^{2 \pi} \int_{0}^{\infty} \delta\left(X-C_{0}(\theta) y^{1 / 2}\right) \frac{d y d \theta}{y^{2}} \\
& =\frac{3}{\pi^{2} X^{3}} \int_{0}^{2 \pi} C_{0}(\theta)^{2} d \theta .
\end{aligned}
$$

So $P(X) \sim P_{1}(X)$ for large $X$, and the proof of Theorem Theorem 1 is complete. 


\section{References}

[1] E. J. Martinec, "Defects, decay, and dissipated states," arXiv:hep-th/0210231.

[2] M. Headrick, S. Minwalla and T. Takayanagi, "Closed string tachyon condensation: An overview," arXiv:hep-th/0405064.

[3] L. Cornalba and M. S. Costa, "Time-dependent orbifolds and string cosmology," Fortsch. Phys. 52, 145 (2004) arXiv:hep-th/0310099].

[4] G. W. Moore, "Les Houches lectures on strings and arithmetic," arXiv:hep-th/0401049.

[5] F. Dowker, J. P. Gauntlett, D. A. Kastor and J. H. Traschen, "Pair creation of dilaton black holes," Phys. Rev. D 49, 2909 (1994) [arXiv:hep-th/9309075.

[6] F. Dowker, J. P. Gauntlett, S. B. Giddings and G. T. Horowitz, "On pair creation of extremal black holes and Kaluza-Klein monopoles," Phys. Rev. D 50, 2662 (1994) arXiv:hep-th/9312172.

[7] F. Dowker, J. P. Gauntlett, G. W. Gibbons and G. T. Horowitz, "The Decay of magnetic fields in Kaluza-Klein theory," Phys. Rev. D 52, 6929 (1995) arXiv:hepth/9507143].

[8] F. Dowker, J. P. Gauntlett, G. W. Gibbons and G. T. Horowitz, "Nucleation of P-Branes and Fundamental Strings," Phys. Rev. D 53, 7115 (1996) arXiv:hepth/9512154].

[9] J. G. Russo and A. A. Tseytlin, "Magnetic flux tube models in superstring theory," Nucl. Phys. B 461, 131 (1996) arXiv:hep-th/9508068.

[10] J. G. Russo and A. A. Tseytlin, "Magnetic backgrounds and tachyonic instabilities in closed superstring theory and M-theory," Nucl. Phys. B 611, 93 (2001) arXiv:hepth/0104238].

[11] J. R. David, M. Gutperle, M. Headrick and S. Minwalla, "Closed string tachyon condensation on twisted circles," JHEP 0202, 041 (2002) arXiv:hep-th/0111212.

[12] J. Cassels, An Introduction to Diophantine Approximation, Cam. Univ. Press 1957.

[13] G. Hardy and E. Wright, An Introduction to the Theory of Numbers, Oxford Univ. Press 1979.

[14] A. Khinchin, Continued Fractions, Univ. of Chicago Press, 1964.

[15] W.M. Schmidt, Diophantine Approximation, Springer-Verlag, LNM 785; Diophantine approximations and diophantine equations, Springer-Verlag LNM 1467.

[16] See the article by Yoccoz in C. Itzykson, J.-M. Luck, P. Moussa, and M. Waldschmidt, eds. From Number Theory to Physics, Springer Verlag, 1995.

[17] B. Kol, "On 6d *gauge* theories with irrational theta angle," JHEP 9911, 017 (1999) arXiv:hep-th/9711017.

[18] S. Elitzur, B. Pioline and E. Rabinovici, "On the short-distance structure of irrational non-commutative gauge JHEP 0010, 011 (2000) [arXiv:hep-th/0009009]. 
[19] C. S. Chan, A. Hashimoto and H. Verlinde, "Duality cascade and oblique phases in non-commutative open string theory," JHEP 0109, 034 (2001) arXiv:hep-th/0107215.

[20] J. A. Harvey, D. Kutasov, E. J. Martinec and G. Moore, "Localized tachyons and RG flows," arXiv:hep-th/0111154.

[21] R. Dijkgraaf and E. Verlinde, "Modular Invariance And The Fusion Algebra," Nucl. Phys. Proc. Suppl. 5B, 87 (1988).

[22] J. A. Harvey, S. Kachru, G. W. Moore and E. Silverstein, "Tension is dimension," JHEP 0003, 001 (2000) arXiv:hep-th/9909072.

[23] D. Kutasov and N. Seiberg, "Number Of Degrees Of Freedom, Density Of States And Tachyons In String Theory Nucl. Phys. B 358, 600 (1991).

[24] D. Kutasov, "Some properties of (non)critical strings," arXiv:hep-th/9110041.

[25] C. Angelantonj, E. Dudas, and J. Mourad, "Orientifolds of String Theory Melvin backgrounds," hep-th/0205096.

[26] N.B. Slater, "Gaps and steps for the sequence $n \theta \bmod 1, "$ Proc. Cambridge Philos. Soc. 63(1967) 1115-1123.

[27] H. Davenport, Analytic Methods for Diophantine Equations and Diophantine Inequalities (Ann Arbor Publ., 1962), page 13ff.

[28] E. Artin, "Ein mechanisches System mit quasiergodischen Bahnen," Collected works, p. 499.

[29] C. Series, The modular surface and continued fractions, J. London. Math. Soc. 31 (1985) 69-80.

[30] H. Liu, G. Moore and N. Seiberg, "Strings in time-dependent orbifolds," JHEP 0210, 031 (2002) arXiv:hep-th/0206182.

[31] M. Pollicott and H. Weiss, "Multifractal analysis of Lyapunov exponent for continued fraction and Manneville-Pomeau transformations and applications to Diophantine approximation," Commun. Math. Phys. 207(1999)145.

[32] M. Marcolli, "Modular curves, C* algebras, and chaotic cosmology," arXiv:mathph/0312035; M. Marcolli, "Limiting modular symbols and the Lyapunov spectrum," arXiv:math.NT/0111093; Y. Manin and M. Marcolli, "Continued fractions, modular symbols, and non-commutative geometry," arXiv:math.NT/0102006.

[33] D.R. Hofstadter, "Energy levels and wave functions of Bloch electrons in rational and irrational magnetic fields," Phys. Rev. B14 (1976) 2239.

[34] M. S. Costa and M. Gutperle, "The Kaluza-Klein Melvin solution in M-theory," JHEP 0103, 027 (2001) arXiv:hep-th/0012072.

[35] M. Gutperle and A. Strominger, "Fluxbranes in string theory," JHEP 0106, 035 (2001) arXiv:hep-th/0104136.

[36] J. Oxtoby, Measure and Category, Springer-Verlag, GTM vol. 2.

[37] J., Marklof, "The $n$-point correlations between values of a linear form, with an appendix by Z. Rudnick," Ergod. Th. Dyn. Sys. 20 (2000) 1127-1172. 